\title{
Comparison of Microscopic Determination and Rapid Diagnostic Tests (RDTs) in the Detection of Plasmodium Infection
}

\author{
Onyeka Linda Anagu, Moses Nkechukwu Ikegbunam*, Chinelo Kene Unachukwu, \\ Ogwaluonye Uchenna C, Charles Okey Esimone \\ Department of Pharmaceutical Microbiology and Biotechnology, Faculty of Pharmaceutical Sciences, Nnamdi \\ Azikiwe University, Awka, Nigeria \\ Email: ${ }^{*}$ mn.ikegbunam@unizik.edu.ng
}

Received 9 July 2015; accepted 21 August 2015; published 24 August 2015

Copyright (C) 2015 by authors and Scientific Research Publishing Inc.

This work is licensed under the Creative Commons Attribution International License (CC BY).

http://creativecommons.org/licenses/by/4.0/

(c) (i) Open Access

\begin{abstract}
Malaria infection is the most common diagnosis made in Africa. Efficient diagnosis of malaria parasite is very vital for treatment of malaria infection. The efficacy of rapid diagnostic tests (RDTs) in comparison to microscopy, the gold standard, in the diagnosis of malaria in Nigeria has not been fully ascertained. This study compared the sensitivity, specificity and predictive values of RDTs available in Nigeria market with microscopy. Two RDT kits were used and their results were compared with the gold standard, microscopy using thick and thin blood films (TBF and tBF). TBF had sensitivity of $85 \%$, specificity of $30 \%$, positive predictive value (PPV) of $55.2 \%$, and negative predictive value (NPV) of $66.6 \%$; tBF had sensitivity of $80 \%$, specificity of $35 \%$, positive predictive value (PPV) of 55.2\%, and negative predictive value (NPV) of 63.6\%. Among the RDTs, Care Start $\mathrm{HRP}_{2}$ kit had sensitivity of $65 \%$, specificity of $50 \%$, positive predictive value (PPV) of $56.5 \%$, and negative predictive value (NPV) of 59\% while SD Bioline kit had sensitivity of $55 \%$, specificity of $65 \%$, PPV of $61 \%$, and NPV of $59 \%$. It can thus be inferred that rapid diagnostic test kits are not as sensitive as microscopy in diagnosis of malaria parasite, but they are more accurate and are thus suitable alternatives to microscopy.
\end{abstract}

Keywords

Plasmodium falciparium, Microscopy, Rapid Diagnostic Tests

\section{Introduction}

Over-prescription and under-prescription of antimalarials are basically attributed to false-positive or false-nega-

\footnotetext{
"Corresponding author.
}

How to cite this paper: Anagu, O.L., Ikegbunam, M.N., Unachukwu, C.K., Uchenna C, O. and Esimone, C.O. (2015) Comparison of Microscopic Determination and Rapid Diagnostic Tests (RDTs) in the Detection of Plasmodium Infection. Advances in Microbiology, 5, 604-609. http://dx.doi.org/10.4236/aim.2015.58063 
tive laboratory test results obtained in the diagnosis of malaria. In practice however, malaria is often diagnosed solely on the basis of clinical symptoms, irrespective of the outcome of laboratory testing, because most government and teaching hospitals still rely on the time-consuming microscopy in the laboratory diagnosis of malaria. The turnaround time in the testing of malaria using microscopy can be up to $24 \mathrm{hr}$, prompting clinicians to make diagnosis retrospectively [1].

The liver cycle in the life cycle of the malaria parasite ends when the mature schizont ruptures and releases the merozoites into the sinusoids of the liver. The merozoites are then discharged into the circulation. Released merozoites can only invade a red blood cell, thus beginning the erythrocytic stage. The erythrocytic stage of malaria parasites has several important implications in clinical practice. First, this is the only stage causing the complex and varying spectrum of symptoms (fever, nausea, chills, vomiting, headache, fatigue and muscular aches) characterizing the disease in humans. Secondly, the recognition of parasites in the blood of a patient allows the laboratory diagnosis of the infection and the differentiation of the various species as the causal agent [2].

The merozoites released from the liver recognize, attach, and enter the red blood cells by multiple receptor-ligand interactions in as little as 60 seconds. This quick disappearance from the circulation into the red blood cells minimizes the exposure of the antigens on the surface of the parasites, thereby protecting these parasite forms from the host immune response [3] [4]. This also means that the parasite (merozoites) is usually visible in the red blood cells.

Microscopy is the reference/gold standard for the laboratory diagnosis of malaria parasite but its turnaround time is much more than that of RDT and it requires adequate training. RDTs are alternative diagnostic methods because they are quick and easy to carry out. They also require little or no training to perform.

RDTs are principally based on the detection of malaria antigens (Histidine Rich Protein (HRP2), parasite Lactate Dehydrogenase (pLDH), Aldolase enzyme) [5] from peripheral blood using monoclonal antibodies prepared against this malaria antigen target and conjugated to either a liposome containing selenium dye or gold particles in a mobile phase. A second or third capture monoclonal antibody applied to a strip of nitrocellulose acts as the immobile phase. The strip enables the labeled antigen to be captured by the monoclonal anti-body of the mobile phase, thus providing a visible coloured thick line. Incorporation of a labeled goat anti-mouse antibody capture ensures that the system is controlled for migration [6].

This study aimed to compare the performance of Care Start $\mathrm{HRP}_{2}$ and SD Bioline RDT kits with light microscopy in the diagnosis of malaria in symptomatic patients and the detection of malaria parasite in asymptomatic patients in Anambra, Nigeria.

\section{Materials and Methods}

\subsection{Study Area}

The study area is Agulu and Awka South in Anambra State located in the South-East region of Nigeria.

\subsection{Ethics Statements}

Ethical Committee of Anambra State University Teaching Hospital, Amaku, Anambra State, Nigeria approved the study Protocol. Written informed consent was obtained from the adult participants and guardians of child participants.

\subsection{Test Samples}

This consisted blood samples from asymptomatic individuals who have not taken any antimalarial for less than 7 days prior to blood donation (from Faculty of Pharmaceutical Sciences, Nnamdi Azikiwe University, Awka) and symptomatic patients (at the Anambra State University Teaching Hospital, Amaku, Awka) volunteers. Anambra State University Teaching Hospital, Amaku is one of the two teaching hospitals in Anambra State. Patients that usually go there for medical attention are majorly from Awka and other neighboring towns.

\subsection{Sample Size}

A total of 20 blood samples each were collected from both symptomatic and asymptomatic patients. 


\subsection{Clinical Diagnosis}

Clinical diagnosis based on fever (temperature $>37.5^{\circ} \mathrm{C}$ ) and/or history of fever, and other symptoms including; headache, joint pains, body weakness, cough, diarrhea, loss of appetite/refusal of feeds, abdominal pain, and generalized body weakness was carried out by physicians at the outpatient department of the hospital.

\subsection{Light Microscopy}

This was done using standard procedures as proposed by Chessbrough M, 2009. Briefly, blood samples were collected into EDTA bottles. Thick and thin films were made from the blood samples; the films were stained with $10 \%$ Geimsa stain (pH 7.2) for 10 minutes and examined under the microscope (model number: BINOCxi, Micron International, India) using $\times 1000$ magnification. Positive findings were graded on the thick smear using the "plus" system scale: + (1 - 10 parasites per 100 fields), ++ (11 - 100 parasites per 100 fields), $+++(1-10$ parasites per field), ++++ (more than 10 parasites in every field) and for the thin smear by calculating the percentage parasitemia [7].

\subsection{Rapid Diagnostic Test}

The blood samples from the 20 symptomatic and 20 asymptomatic patients were tested using the SD Bioline and Care Start $\mathrm{HRP}_{2}$ kits. SD Bioline (CAT No: 05FK90, Standard Diagnostics Inc., Korea) and Care Start $\mathrm{HRP}_{2}$ kits (CAT No: G0141, Access Bio Inc., USA) are lateral flow immunochromatographic antigen detection tests kits in a cassette form. The testing was carried out according to manufacturer's instructions. Negative result is indicated by the presence of a single line, while a positive result is indicated by two bands in the strip.

\subsection{Data Analysis}

The sensitivity, specificity, and predictive values of each of the three test methods were calculated by comparing to a standard. The standard was the absence or presence of clinical symptoms for malaria parasites as determined by a physician. This gives the standard $100 \%$ hypothetical sensitivity, specificity, and positive and negative predictive values. The sensitivity, specificity, and predictive values of each of the four methods; microscopy by thick blood film, thin blood film, and rapid diagnostic test by SD Bioline and Care Start $\mathrm{HRP}_{2}$ kits were then calculated using the formulas below.

$$
\begin{gathered}
\text { Sensitivity }=\frac{T P}{T P+F N} \times 100 \\
\text { Specificity }=\frac{T N}{T N+F P} \times 100 \\
P P V=\frac{T P}{T P+F P} \times 100 \\
N P V=\frac{T N}{T N+F N} \times 100
\end{gathered}
$$

where $T P=$ true positive, $F P=$ false positive, $T N=$ true negative, and $F N=$ false negative. Sensitivity was defined as the probability that a truly infected individual will test positive and specificity as the probability that a truly uninfected individual will test negative.

\section{Results}

\subsection{Prevalence of Malaria Infections as Measured by the Microscopy and RDT}

The diagnostic value of Microscopy (thick and thin blood film) and RDTs in the detection of malaria parasites in South-east Nigeria was compared among symptomatic patients. Its ability to detect sub patent infection was also compared in asymptomatic patients.

The result of microscopy is shown in Table 1. For the thick blood film (TBF), 3 asymptomatic patient result had ++, 5 symptomatic had ++, while 1 symptomatic had +++ result. For the thin blood film (tBF), the average 
percentage parasitemia was used to verify the presence of malaria parasite.17 (85\%) patients were positive for malaria by TBF and $16(80 \%)$ by tBF. The parasite was detected in $14(70 \%)$ asymptomatic individuals by TBF and 13 (65\%) by tBF.

The rapid diagnostic test using SD Bioline and Care Start $\mathrm{HRP}_{2}$ kits are shown in Table 2. 13 (65\%) patients were positive for malaria by Care Start kit (CSK) and 11 (55\%) by SD Bioline kit (SBK). The parasite was detected in 10 (50\%) asyptomatic individuals by CSK and 7 (35\%) by SBK.

\subsection{Comparison of Microscopy and RDT}

In comparing the diagnostic methods with the standard method, TBF had sensitivity of $85 \%$, specificity of $30 \%$, positive predictive value (PPV) of 55.2\%, and negative predictive value (NPV) of $66.6 \%$, tBF had sensitivity of $80 \%$, specificity of $35 \%$, positive predictive value (PPV) of $55.2 \%$, and negative predictive value (NPV) of $63.6 \%$. Among the RDTs, CSK has sensitivity of $65 \%$, specificity of $50 \%$, positive predictive value (PPV) of $56.5 \%$, and negative predictive value (NPV) of $59 \%$ while SBK had sensitivity of $55 \%$, specificity of $65 \%$, PPV of $61 \%$, and NPV of $59 \%$ (Table 3 ).

\section{Discussion}

Majority of hospitals in Nigeria still prescribe drugs based on symptomatic presentation and as such clinical diagnosis has become our gold standard. Precision in medical therapy comes about where a second opinion is sought for confirmation of the disease condition. Some illnesses have similar presentation. This study thus tells us that $85 \%$ of tentative diagnosis for malaria is correct when using the most sensitive diagnostic tool, microscopy, using thick blood film, while the remaining $15 \%$ suffers from other symptom related illnesses.

There are four principal methods for diagnosis of malaria: symptomatic, microscopy, antigen test and molecular

Table 1. Summary of result for microscopy.

\begin{tabular}{ccccc}
\multicolumn{2}{c}{ Thick blood film } & \multicolumn{2}{c}{ Thin blood film } \\
\cline { 2 - 5 }+ & Asymptomatic & symptomatic & Asymptomatic & symptomatic \\
\hline- & 14 & 17 & 13 & 16 \\
\hline
\end{tabular}

Table 2. Summary of result for RDT.

\begin{tabular}{ccccc}
\hline & \multicolumn{2}{c}{ Care Start kit } & \multicolumn{2}{c}{ SD Bioline kit } \\
\cline { 2 - 5 }+ & Asymptomatic & Symptomatic & Asymptomatic & Symptomatic \\
\hline+ & 10 & 13 & 7 & 11 \\
\hline
\end{tabular}

Table 3. Test of performance.

\begin{tabular}{ccccc} 
& Sensitivity (\%) & Specificity (\%) & PPV (\%) & NPV (\%) \\
\hline & & RAPID DIAGNOSTIC TEST & & 59 \\
Care start & 65 & 50 & 56.5 & 59 \\
SD Bioline & 55 & 65 & 61 & 66.6 \\
Thick film & 85 & MICROSCOPY & 55.2 & 63.6 \\
\hline
\end{tabular}

PPV—positive predictive value; NPV—negative predictive value. 
methods. Symptomatic (use of clinical symptoms) method in most cases is the first and only step in malaria diagnosis especially in low to most middle income countries. But in developed countries symptomatic diagnosis is confirmed using one or more of the other methods before treatment is administered.

Microscopic examination of TBF (85\%) was able to detect more parasites in both symptomatic patients and patients with sub-patent infections (the asymptomatic individuals) than tBF (80\%), CSK (65\%) and SBK (55\%). This is in agreement with previous works [8] [9]. Sensitivity of RDTs increases with an increase in parasitaemia with a limiting value of 100 parasites $/ \mu \mathrm{L}$, but it is expected that any RDT used for malaria diagnosis should have a high sensitivity (95\%) and specificity (97\%) [10] [11]. The sensitivity of RDT is lower than that reported in this study [9] but similar to that reported in this study [12].

The specificity of microscopy as expected is lower (30\% \& 35\%) than RDTs (50\% and 65\%), but the higher the specificity the more accurate the diagnostic value. It was only able to detect $30 \%$ - 35\% of asymptomatic patients as malaria free. This is probably due to the presence of sub-patent infection which does not manifest as an illness. RDT was more specific than microscopy as it only detects malaria when the antigen level is at a particular limit due to the present of a limiting number of parasites in the blood. However, its low specificity could be due to self medication taken by patients before they present to the hospital for treatment.

The predictive values of the various diagnostic tests show that there is a slightly greater chance than average that a positive result is actually positive and a negative result means that the patient has no malaria infection, as they all had PPV and NPV as 55.2\% and $66.6 \%$ for TBF, $55.2 \%$ and $63.6 \%$ for tBF, $56.5 \%$ and $59 \%$ for CSK, and $61 \%$ and $59 \%$ for SBK. These values do not agree with previous studies [8]-[10] [12].

False positive results in RDT can be due to factors such as persistence of HRP2 antigen in patient blood weeks after a successful treatment [13]; plasmodia gametocyte also produces pLDH thus test could be positive despite clearance of asexual forms of parasite [14]. It could also be due to interaction with rheumatoid factor found in patients with rheumatoid arthritis [15]-[17]. While false negative results can occur due to presence of anti-HRP2 antibodies in humans [18], it also could be attributed to the storage conditions of RDT kits after manufacture till it gets to the laboratory.

False positive results in microscopy can result from inadequately trained staff that report artifacts as positive result. False negative results in microscopy may be due to inadequately trained staff that cannot recognize the parasite or stain the test specimen properly.

RDTs are rapid and simple to perform and to interpret, but have limitations in detecting the presence of malaria parasite in low parasitemia. RDT should be carefully evaluated for proper quality and storage; otherwise implementation of RDTs may not be beneficial. Microscopy still remains the gold standard for detecting malaria parasite although it is less specific than RDT. The use of symptomatic method has led to an increase in the misdiagnosis and inadvertently misuse of antimalarial which may eventually contribute to drug resistance. Some underlying missed illness can progress to become complicated due to delay in drug administration. Still because of the time-consuming nature of microscopy, to prevent malaria diagnosis to be based solely on clinical investigation, RDTs can be used as they are even more accurate. Those few missed diagnosis that cannot be detected by the kits can be detected with microscopy since their viral load is low and there is little chance of complications developing, especially in older patients.

The sample size used in this study could hinder our drawing adequate conclusions from our findings and may reduce the accuracy of this study when it is being applied to the large populace.

\section{Acknowledgements}

We thank all the patients who participated in this study. In addition, we are also grateful to Pharm. Malachy Ugwu of the department of Pharmaceutical microbiology and Biotechnology, Nnamdi Azikiwe University, Awka. Dr. Sir Emma Emelummadu, Dr. Ifeanyi Ezeobi, Mbidoaka Joycee Iheakachi and the entire staff of Sefton Specialist Hospital Awka.

\section{Author Summary}

Our study was informed by the observation that Rapid Diagnostic Test kits (RDTs) have become widely used but predominantly in private hospitals and laboratory. Their use in government owned health institutions is limited in malaria diagnosis, where clinical diagnosis only is encouraged prior to administration of drugs and use of the time consuming microscopy is made retrospectively. We decided to compare microscopy and RDTs in 
malaria diagnosis so as to provide a basis for adoption of the use RDTs in lieu of microscopy in government hospitals in Nigeria. We used microscopic examination of thick and thin blood film and rapid diagnostic test kits to detect the presence of malaria parasite in symptomatic and asymptomatic individuals. We then compared the performance of these diagnostic testing procedures by calculating their sensitivity, specificity and predictive values in order to find out which of them is more sensitive and accurate. Our study indicates that it is okay to use RDTs even though it is less sensitive, as it is more accurate. The few clinically suspected cases that are not confirmed by RDTs due to low parasitemia can always be confirmed using microscopy. It may even aid to diagnose any missed illnesses.

\section{References}

[1] Ameh, J., Ahmad, R.M., Ekeh, N., Linga, P., Mangoro, Z., et al. (2012) Laboratory Diagnosis of Malaria: Comparing Giemsa Stained Thick Blood Films with Rapid Diagnostic Test (RDT) in an Endemic Setting in North-West Nigeria. Journal of Medical Laboratory and Diagnosis, 3, 10-15. http://dx.doi.org/10.5897/JMLD12.001

[2] Graham, P.C.C. (1996) Malaria Parasites and Other Haemosporida. Blackwell Scientific Publication, Oxford.

[3] Silviee, O., Mata, M.M., Matuschewski, K. and Prudencio, M. (2008) Interactions of the Parasite and Its Mammalian Host. Current Opinion in Microbiology, 11, 352-359. http://dx.doi.org/10.1016/j.mib.2008.06.005

[4] Cowman, A.F. and Crabb, B. (2006) Invasion of Red Blood Cells by Malaria Parasite. Cell, 124, 755-766. http://dx.doi.org/10.1016/j.cell.2006.02.006

[5] Murray, C.K., Bell, D., Grasser, R.A. and Wongsrichanalai, C. (2003) Rapid Diagnostic Testing for Malaria. Tropical Medicine \& International Health, 8, 876-883. http://dx.doi.org/10.1046/j.1365-3156.2003.01115.x

[6] Piper, R, Vanderjagt, L., Holbrook, J.J. and Makler, M. (1996) Malaria Lactate Dehydrogenase; Target for Diagnosis and Drug Development. Annals of Tropical Medicine \& Parasitology, 90, 433.

[7] Chessebrough, M. (2009) District Laboratory Practice in Tropical Countries.

[8] Sun, X.D., Tambo, E., Wei, C., Chen, Z.B., Deng, Y., Wang, J., Wang, J.Z. and Zhou, X.N. (2013) Diagnostic Performance of CareStart ${ }^{\mathrm{TM}}$ Malaria HRP2/pLDH (Pf/pan) Combo Test versus Standard Microscopy on Falciparum and Vivax Malaria between China-Myanmar Endemic Borders. Malaria Journal, 12, 6. http://dx.doi.org/10.1186/1475-2875-12-6

[9] Sheyin, Z. and Bigwan, I.E. (2013) Comparison of CARE START HRP2 Rapid Malaria Test with Light Microscopy for Guiding Patient's Treatment of Fever in Nigeria Endemic Areas. Journal of Medicine and Medical Sciences, 4, 353-356.

[10] Cheng, Q., Cloonan, N., Fischer, K., et al. (1998) Stevor and Rif Are Plasmodium falciparum Multicopy Gene Families Which Potentially Encode Variant Antigens. Molecular and Biochemical Parasitology, 97, 161-176. http://dx.doi.org/10.1016/S0166-6851(98)00144-3

[11] WHO (2003) Malaria Rapid Diagnosis: Making It Work. World Health Organization, Manila.

[12] Ojurongbe, O., Adegbosin, O.O., Taiwo, S.S., Oyebode, A.T., Olowe, O.A., Ojurongbe, T.A., et al. (2013) Assessment of Clinical Diagnosis, Microscopy, Rapid Diagnostic Tests, and Polymerase Chain Reaction in the Diagnosis of Plasmodium falciparum in Nigeria. Malaria Diagnosis and Treatment, 2013, Article ID: 308069.

[13] Moody, A., Hunt-Cooke, A., Gabbett, E. and Chiodini, P. (2000) Performance of the OptiMAL Malaria Antigen Capture Dipstick for Malaria Diagnosis and Treatment Monitoring at the Hospital for Tropical Diseases, London. British Journal of Haematology, 109, 891-894. http://dx.doi.org/10.1046/j.1365-2141.2000.01974.x

[14] Miller, R.S., McDaniel, P. and Wongsrichanalai, C. (2001) Following the Course of Malaria Treatment by Detecting Parasite Lactate Dehydrogenase Enzyme. British Journal of Haematology, 113, 558-559. http://dx.doi.org/10.1046/j.1365-2141.2001.02782.x

[15] Humar, A., Ohrt, C., Harrignton, M.A., Pillai, D. and Kain, K.C. (1997) Parasight F Test Compared with the Polymerase Chain Reaction and Microscopy for the Diagnosis of Plasmodium falciparum Malaria in Travelers.

[16] Mishra, B., Samantaray, J.C., Kumar, A. and Mirdha, B.R. (1999) Study of False Positivity of Two Rapid Antigen Detection Tests for Diagnosis of Plasmodium falciparum Malaria. Journal of Clinical Microbiology, 37, 1233.

[17] Laferl, H., Kandel, K. and Pichler, H. (1997) False Positive Dipstick Test for Malaria. New England Journal of Medicine, 337, 1635-1636. http://dx.doi.org/10.1056/NEJM199711273372219

[18] Biswas, S., Tomar, D. and Rao, D.N. (2005) Investigation of the Kinetics of Histidine-Rich Protein 2 and of the Antibody Responses to This Antigen, in a Group of Malaria Patients from India. Annals of Tropical Medicine \& Parasitology, 99, 553-562. http://dx.doi.org/10.1179/136485905X51463 